\title{
Safe and easy in vitro evaluation of tmRNA-SmpB-mediated trans-translation from ESKAPE pathogenic bacteria
}

Marion Thépaut ${ }^{1,2 \dagger}$, Rodrigo Campos-Silva ${ }^{1,3 \dagger}$, Eva Renard ${ }^{4}$, Frédérique Barloy-Hubler ${ }^{1}$, Eric Ennifar $^{4}$, Daniel Boujard ${ }^{1}$, and Reynald Gillet ${ }^{1 *}$

${ }^{1}$ Univ. Rennes, CNRS, Institut de Génétique et Développement de Rennes (IGDR) UMR 6290, Rennes, France

${ }^{2}$ SATT Ouest-Valorisation, Rennes, France

3 Programa de Pós-Graduação em Ciências Farmacêuticas, Faculdade de Farmácia, Universidade Federal do Rio Grande do Sul, Porto Alegre, Brazil

${ }^{4}$ Architecture et Réactivité de l'ARN - CNRS UPR 9002, Institut de Biologie Moléculaire et Cellulaire, Université de Strasbourg, Strasbourg, France

†Equal contribution.

* Corresponding author: Reynald Gillet

E-mail: reynald.gillet@univ-rennes1.fr

Running title: Evaluation of trans-translation in ESKAPE bacteria

Keywords: Trans-translation, tmRNA, ESKAPE, antibiotics, HTS, ribosome

\section{Abstract}

In bacteria, trans-translation is the major quality control system for rescuing stalled ribosomes.

It is mediated by tmRNA, a hybrid RNA with properties of both a tRNA and a mRNA, and the small protein SmpB. Because trans-translation is absent in eukaryotes but necessary for bacterial fitness or survival, it is a promising target for the development of novel antibiotics. To facilitate screening of chemical libraries, various reliable in vitro and in vivo systems have been created for assessing trans-translational activity. However, the aim of the current work was to permit the safe and easy in vitro evaluation of trans-translation from pathogenic bacteria, which 
are obviously the ones we should be targeting. Based on green fluorescent protein (GFP) reassembly during active trans-translation, we have created a cell-free assay adapted to the rapid evaluation of trans-translation in ESKAPE bacteria, with 24 different possible combinations. It can be used for easy high-throughput screening of chemical compounds as well as for exploring the mechanism of trans-translation in these pathogens.

\section{Introduction}

The World Health Organization (WHO) designated six 'ESKAPE' pathogens (Enterococcus faecium, Staphylococcus aureus, Klebsiella pneumoniae, Acinetobacter baumannii, Pseudomonas aeruginosa, and Enterobacter spp.) as critical targets for drug discovery (Rice et al., 2008; Tacconelli and Magrini, 2017). Indeed, these bacteria are the leading cause of nosocomial infections throughout the world, and most are multidrug-resistant isolates (Santajit and Indrawattana, 2016). The WHO recommendation is to focus specifically on the discovery and development of new antibiotics that are active against multidrug- and extensively drugresistant ESKAPE bacteria. However, the hazardous nature of these pathogens makes it highly challenging to develop high-throughput screening methods for identifying and evaluating any new antimicrobial agents for future clinical use. To aid in this, the molecular process to be targeted must first be identified, and ideally this process should be: $i$ ) conserved among all pathogenic ESKAPE bacteria; ii) indispensable to bacterial survival or at least its fitness; iii) sufficiently variable that different species can be distinguished from each other; $i v$ ) absent in eukaryotes; v) not targeted by current antibiotics; vi) unrelated to existing resistance mechanisms; and finally vii) reproducible in non-hazardous in vitro experiments.

In fact, trans-translation appears to be the perfect candidate. This mechanism is the primary bacterial rescue system, allowing for the release of ribosomes stalled on faulty mRNAs that lack stop codons as well as the elimination of these mRNAs and mistranslated peptides. 
The trans-translation process is performed by hybrid transfer-messenger RNA (tmRNA) and its protein partner SmpB (Giudice et al., 2014). Briefly, the tmRNA-SmpB complex recognizes the stalled ribosome and associates with it. In a finely orchestrated ballet, translation then resumes on tmRNA's internal mRNA-like domain (MLD), which encodes a specific sequence that is recognized by proteases. This process permits the stalled ribosomes to be recycled, the degradation of the incomplete peptide after its release, and elimination of the problematic nonstop mRNA. Remarkably, genes coding for tmRNA and SmpB have been found in nearly all bacterial genomes, yet not in eukaryotes, with the exception of a very few rare organelles (Hudson and Williams, 2015). Despite high sequence conservation at both the 5'- and 3 '-ends of tmRNA genes, the internal sequences of tmRNA are considerably divergent among different species (Supp. Fig. 1), and this property makes tmRNA a good tool for species identification (Schonhuber et al., 2001). In the same way, despite global structural conservation, variations in $s m p B$ sequences are also considerably divergent among different species (Supp. Fig. 1).

While resolving stalled ribosomal complexes is undoubtedly a matter of life or death (Keiler and Feaga, 2014), trans-translation itself is not always indispensable to bacterial survival. This irregularity was the subject of a long debate until the discovery of backup systems, mechanisms which take over if trans-translation is deficient or overwhelmed. However, even when they are present, these systems are not enough to ensure a steady and prolonged fitness to the cell, as impaired trans-translation is known to result in various phenotypes varying from mild (such as loss of tolerance to multiple antibiotics and stresses) to severe (including lethality or loss of virulence) (Li et al., 2013; Keiler and Feaga, 2014). To date, trans-translation has not been yet exploited for clinical use. In the search for inhibitors specific to the process, initial assays led to the discovery of 1,3,4 oxadiazole molecules (Ramadoss et al., 2013), but their specificity for trans-translation in vivo is still in question (Macé et al., 2017; Brunel et al., 2018). It has been suggested that trans-translation is inhibited 
by pyrazinamide (PZA), a first-line anti-tuberculosis drug (Shi et al., 2011), but it was finally recently shown the action of PZA is entirely independent of trans-translation in M. tuberculosis (Dillon et al., 2017).

Because of its biological properties, transfer of trans-translation into a non-hazardous system that could allow for rapid and easy evaluation of its activity would greatly help in the search for new antibiotics which target this system. While there are routine methods for screening the antimicrobial activity of compounds from chemical libraries, a combination of this primary screening with the specification of a molecular target is much harder to implement (Osterman et al., 2016). An ideal method would allow not just the identification of the targeted cellular process, but also its level of specificity toward a bacterial genus or species. Furthermore, an easy quantitative and rapid analysis of the process should be possible even in small volumes. Reporter assays are the best candidates for efficient initial high-throughput screening (HTS) methods, as they can be quick and automated, as well as quite useful for screening unpurified mixtures of natural extracts (Osterman et al., 2016). Accordingly, we recently used a commercial reconstituted in vitro translation system (PURExpress) to create a reliable in vitro reporter system that detects the E. coli trans-translation activity (Guyomar et al., 2020). This assay, based on reassembling an active "superfolder" Green Fluorescent Protein (sfGFP) after tmRNA tagging (Fig. 1), was designed and validated for the specific in vitro quantification of trans-translation in ESKAPE pathogenic bacteria, and we report on that here.

A

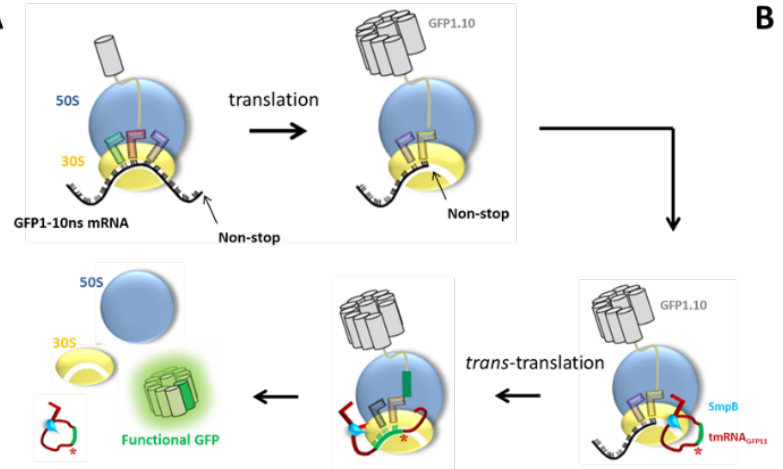

B

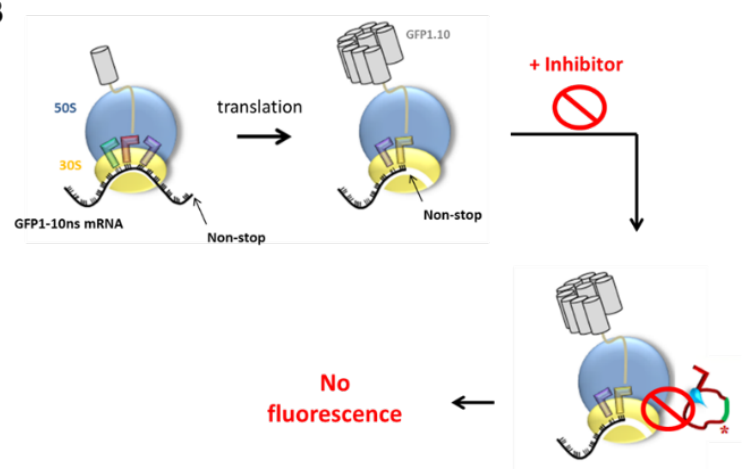

Figure 1. A. Trans-translation of sfGFP1-10 mRNA lacking a stop codon. The tmRNA ${ }_{G F P 11-S m p B}$ 
complex binds to the stalled ribosome, and translation resumes thanks to the tmRNAGFP11 mRNA-like domain (MLD). The MLD encodes the missing eleventh beta-strand of the sfGFP, and the complete sfGFP is released and becomes fluorescent. B. Impairment of the process in the presence of transtranslation inhibitors. The ribosomes stay stalled on the problematic mRNA and fluorescence is impaired.

\section{Results}

\section{Distribution of ArfA, ArfB and RQC in ESKAPE bacterial genomes}

While some bacteria can survive without trans-translation, this is only because of the existence of backup systems, such as the two alternative release factors, ArfA and ArfB or the bacterial ribosome-associated quality control (RQC) mediated by RqcH and RqcP. The Arf mechanisms can be divided in release factor (RF)-dependent and RF-independent mechanisms. ArfA recruits RF2 to hydrolyze the nascent polypeptide chain from the P-site tRNA, while ArfB, a class I release factor homologue, performs hydrolysis itself (Himeno et al., 2015; Müller et al., 2021) On the other hand, $\mathrm{RqcH}$ and $\mathrm{RqcP}$ act in concert to mediate the Ribosome-associated qualitycontrol (RQC) pathway, triggering C-terminal tailing of stalled peptides in the large ribosomal subunit. RqcH belongs to the NEMF family proteins (homolog of the eukaryotic RQC factor Rqc2/NEMF, while RqcP (ribosome quality control P-tRNA, formerly YabO), belongs to the widely distributed S4 RNA-binding family, and is homologous to E. coli heat shock protein 15 (Hsp15) (Lytvynenko et al., 2019; Müller et al., 2021). Depending on backup system status, therefore, the effects of specific inhibitors of trans-translation will vary, from increasing the activity of currently used antibiotics to outright cell death. It was therefore important for us to begin by pinpointing the phylogenetic distribution of ArfA, ArfB and RQC in ESKAPE pathogens. To do this, we investigated the sequences of those rescue factors using a combination of in silico methods including keyword searches, similarity detection, protein domain prediction, ortholog clustering, and synteny analysis. This pipeline was applied to the complete genomic sequences of 1,670 species: $147 \mathrm{E}$. faecium, $473 \mathrm{~S}$. aureus, $465 \mathrm{~K}$. pneumoniae, 188 A. baumannii, 259 P. aeruginosa, and 151 Enterobacter spp. Interestingly, 
among these ESKAPE pathogens, none of the back-up systems were found in A. baumannii while the two Gram-positive bacteria E. faecium and S. aureus displayed RQC only (Table 1).

While we cannot categorically state that no other backup systems exist in these bacteria - see for instance the recent reports on ArfT in Francisella tularensis and BrfA in Bacillus subtilis (Goralski et al., 2018; Shimokawa-Chiba et al., 2019) - we can however suppose that their viability highly depends on trans-translation impairment. On the other hand, we found genes encoding ArfA and/or ArfB in most if not all of the K. pneumonia, P. aeruginosa, and Enterobacter spp. studied. The impairment of trans-translation in these bacteria is probably less severe, therefore, even if it still detrimental to bacterial fitness.

Table 1. Distribution of $\operatorname{arfA}$, $\operatorname{arfB}, r q c H$ and $r q c P$ in ESKAPE bacteria

\begin{tabular}{|l|c|c|c|c|c|}
\hline ESKAPE pathogen & \# of screened genomes & arfA & arfB & rqcH & rqcP \\
\hline Enterococcus faecium & 147 & 0 & 0 & 147 & 147 \\
\hline Staphylococcus aureus & 473 & 0 & 0 & 473 & 473 \\
\hline Klebsiella pneumoniae & 465 & $464+1 \Delta$ & $459+6 \Delta$ & 0 & 0 \\
\hline Acinetobacter baumannii & 188 & 0 & 0 & 0 & 0 \\
\hline Pseudomonas aeruginosa & 259 & 259 & 259 & 0 & 0 \\
\hline Enterobacter spp. & 151 & 151 & $150+3 \Delta$ & 0 & 0 \\
\hline
\end{tabular}

$\Delta$ pseudogenes with frameshift or « in-frame » stop codon.

\section{ESKAPE tmRNA and SmpB production}

To allow for independent monitoring of trans-translation in these six ESKAPE pathogens, we engineered their tmRNAs by replacing their internal MLD with a sequence of 16 amino acids that encodes GFP's eleventh bete-strand (Supp. Table 1). To conserve the tmRNA H5 helix that

146 is instrumental during trans-translation, we also engineered compensatory mutations (Guyomar 147 et al., 2020, and Supp. Fig. 2A, B). Unlike those of the other bacteria, the natural tmRNA 3'ends in E. faecium and S. aureus are not CCA but UUG and UAU, respectively, so these were 
replaced by CCA to ensure correct aminoacylation by E. coli AlaRS (Barends et al., 2000), and

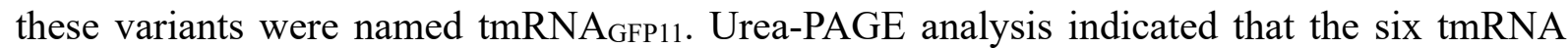
variants were successfully produced at the expected size, without any noticeable degradation or unexpected bands (Supp. Fig. 2D). We started with $10 \mu$ g plasmidic DNA, and the final yields were about $4 \mathrm{nmol}$ of transcribed RNAs for each reaction. The six corresponding SmpB proteins were cloned and produced in vivo in E. coli (see Experimental Procedures). After purification, polyacrylamide gel analysis confirmed the correct size of each protein (Supp. Fig. 2E). The final yields for each ESKAPE SmpB were about half the amount of the E. coli SmpB produced.

\section{ESKAPE trans-translation reactions}

In order to obtain non-productive translation complexes (NTCs) to be targeted by transtranslation, we used a reconstituted cell-free protein synthesis (NEB PURExpress ${ }^{\circledR}$ ) system from E. coli (Shimizu, et al., 2001, Shimizu and Ueda, 2002). By adding a non-stop DNA template, we accumulated stalled ribosomes with the ten first beta-strands of sfGFP stuck in the ribosome exit tunnel (Fig. 1A). When tmRNA ${ }_{\mathrm{GFP} 11}$ and E. coli SmpB are added, the ribosomes are freed and the intensity of the fluorescent signal increases over time while the complete sfGFP protein is produced. A plateau is reached at $\sim 4$ hours of incubation, and the fluorescence remains stable for at least 710 minutes (Fig. 2A, black curve).

In a first set of heterologous experiments, we kept the $E$. coli tmRNA $_{\mathrm{GFP} 11}$, but replaced its SmpB by one from an ESKAPE pathogen. A fluorescent signal was still recovered with each one of the hetero-complexes, albeit at different levels (Fig. 2A). The E. cloacae, S. aureus, and P. aeruginosa SmpBs displayed the lowest signals, less than $30 \%$ of the E. coli control, while the K. pneumoniae SmpB signal was about half the control, and E. faecium and A. baumannii at $80 \%$. This demonstrates that all of the ESKAPE SmpBs are functional and sufficiently conserved to be interchangeable in the presence of $E$. coli tmRNA. While it confirms that SmpB 
174 is highly conserved (Supp. Fig. 1), it also supports the use of this simple system for screening 175 molecules that target SmpB but not tmRNA. Indeed, since SmpB is essential for tmRNA's 176 peptide-tagging activity (Karzai et al., 1999), disrupting SmpB is one of the most promising 177 ways to impair trans-translation. In fact, aptamers that inhibit SmpB functioning were recently 178 shown to trigger strong growth defects in Aeromonas veronii C4 (Liu et al., 2016).

We then performed the experiments the other way around, using the E. coli $\mathrm{SmpB}$ but the tmRNAs from the ESKAPE pathogens. Contrary to the previous experiments, only the heteroduplexes combining E. coli SmpB and the tmRNAs from K. pneumoniae and E. cloacae gave out strong signals, about the same levels as those recovered in the E. coli tmRNA control (Fig. 2B). This is not a surprise since, like E. coli, both $K$. pneumoniae and E. cloacae are Enterobacteriaceae with very similar tmRNA sequences ( $\geq 95 \%$ identity with $E$. coli, see Supp. Fig. 1). The four other bacterial species all produced signals, but at lower levels (about 5 to $20 \%$ of the reference).

We continued by performing homologous experiments, using SmpB and tmRNA $A_{G F P 11}$ from the same ESKAPE pathogen, but this time with E. coli ribosomes (Fig. 2C). Five of the six complexes yielded positive results. Three of these were at high levels ( $\sim 50 \%$ compared to the E. coli reference): K. pneumonia; E. cloacae and E. faecium. The other two were at lower levels (about $5-10 \%$ of the reference): A. baumannii, another Gammaproteobacteria that is relatively close to Enterobacteriaceae; and the Gram-positive $S$. aureus. The Gammaproteobacteria P. aeruginosa did not work at all. 
A

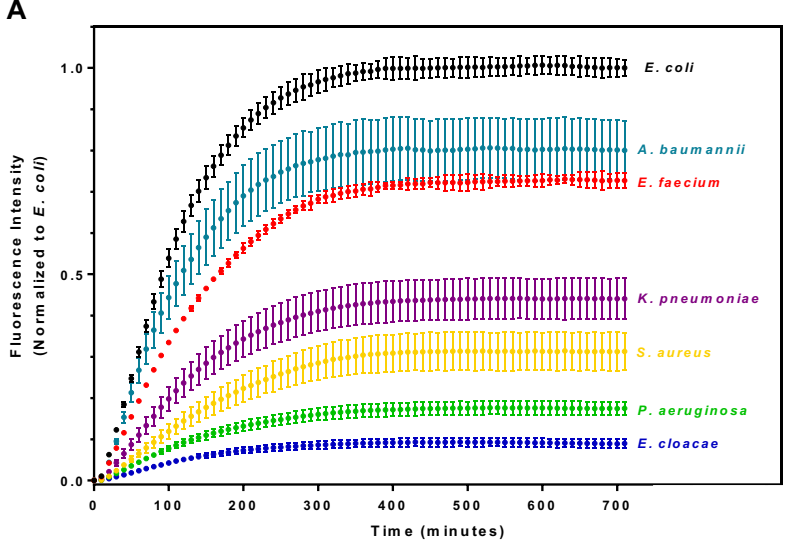

B

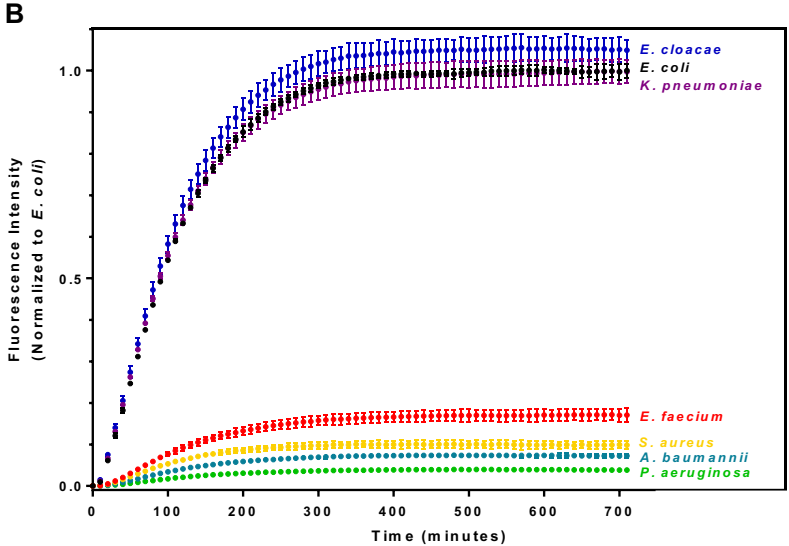

C

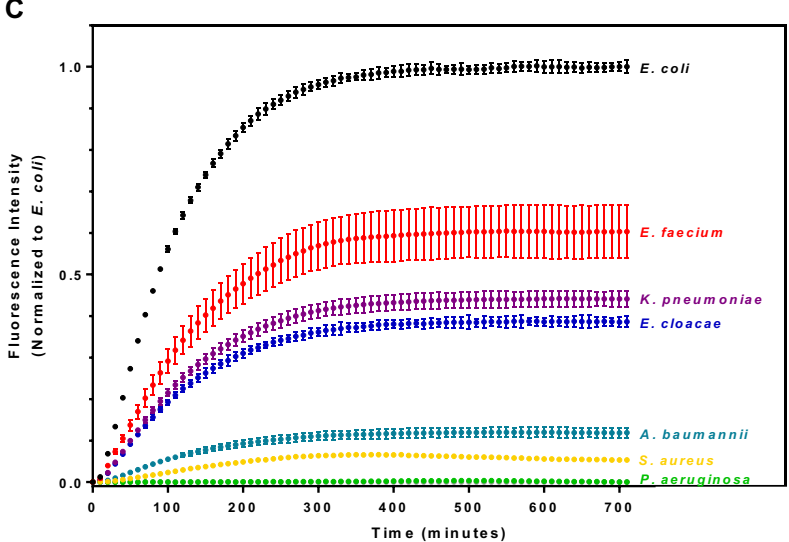

Figure 2: Trans-translation kinetics over time using Escherichia coli ribosomes. Fluorescence increases are directly linked to trans-translation activity. A) Trans-translation assays were done on $E$.

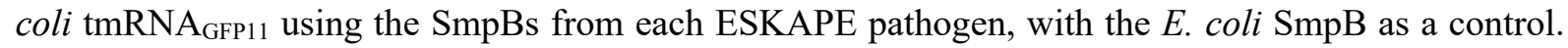

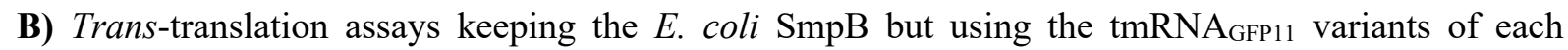
ESKAPE pathogen, with an $E$. coli tmRNA $_{\mathrm{GFP} 11}$ as the control. C) Both SmpB and tmRNA $\mathrm{GFP} 11_{\text {are from }}$ each ESKAPE pathogen, with the $E$. coli $\mathrm{SmpB}$-tmRNA $\mathrm{GFP}_{11}$ as a control. The results are shown as means \pm standard deviation and normalized to the $E$. coli conditions. 

synthesizing full-size sfGFP. For all ribosomes used, a fluorescent signal was recovered, indicating that the ESKAPE ribosomes translate well even if at lower levels (Fig. 3A). The $P$.

A

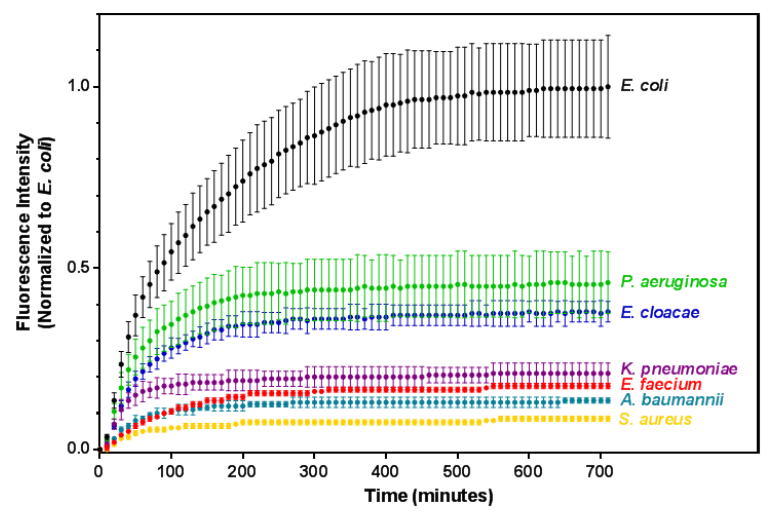

B

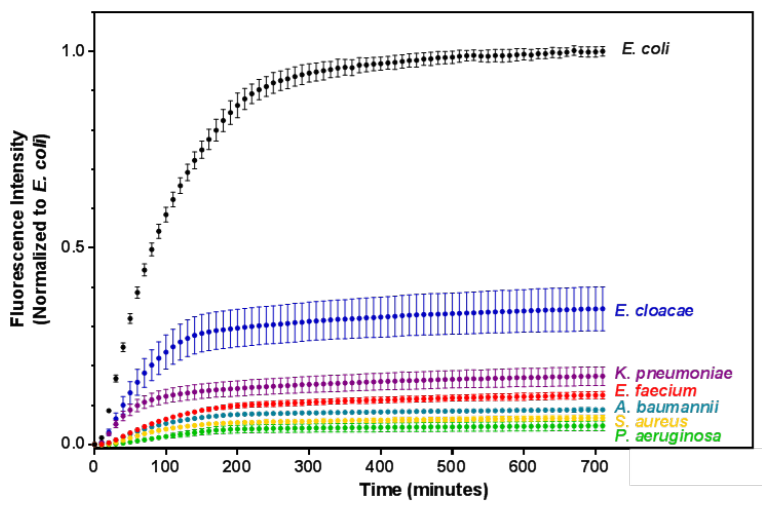

Figure 3. Translation and trans-translation kinetics over time. A) Translation kinetics over time: the increase in fluorescence of full-length GFP (i.e. encompassing the 12 beta-strands) is directly linked to translation. B) Trans-translation kinetics over time using ESKAPE ribosomes. All results are shown as means \pm standard deviation and normalized to $E$. coli.

Despite these rather poor translation rates, fluorescence was easily detected, so we also performed trans-translation experiments using ESKAPE ribosomes (Fig. 3B). The goal was to improve the levels of the trans-translation signals previously recovered, but more importantly to obtain a positive result for $P$. aeruginosa. The results were finally conclusive for that bacteria, which gave a fluorescent signal of $\sim 10 \%$ compared to the control. This positive result could be linked to the quite efficient translation obtained with these ribosomes (Figure 3A). On the other hand, the trans-translation levels of the other bacteria did not improve, and were even lower in 
$225 S$. aureus. This could be due to the fact that the PURExpress system is based on only E. coli

226

227 translation factors, and their low count limits their handling of canonical translation (see Fig. 3A) or specific tmRNAs (e.g. tmRNA aminoacylation by E. coli AlaRS or tmRNA-SmpB transport by E. coli EF-Tu-GTP). However, and since our goal was to detect variations of fluorescence after drug treatment within each of the ESKAPE species, rather than comparing the strains between each other, it was important to get a correct and satisfactory signal for each one of the strains individually. Towards this aim, we decided to use a more sensitive spectrophotometer, ie Synergy HTX from BioteKC. We adjusted the spectrophotometer gain function in order to ensure optimal detection of GFP fluorescence without saturation and applied the technique to the homologous systems (tmRNA-SmpB and ribosomes from the same ESKAPE), the ones that are the most interesting for developing new inhibitors. The data obtained were finally conclusive, within a range of 20000-90000AU for translation as well as for trans-translation, allowing for an accurate internal control in case of inhibition (Supp. Fig. $3)$.

\section{Discussion}

Here we describe the use of GFP as a reporter for safe measurement of the trans-translation activity of the six ESKAPE systems in a cell-free protein synthesis system. The various combinations we evaluated (four for each ESKAPE pathogen) have yielded different interesting strategies for the disruption of trans-translation (Fig. 4). 

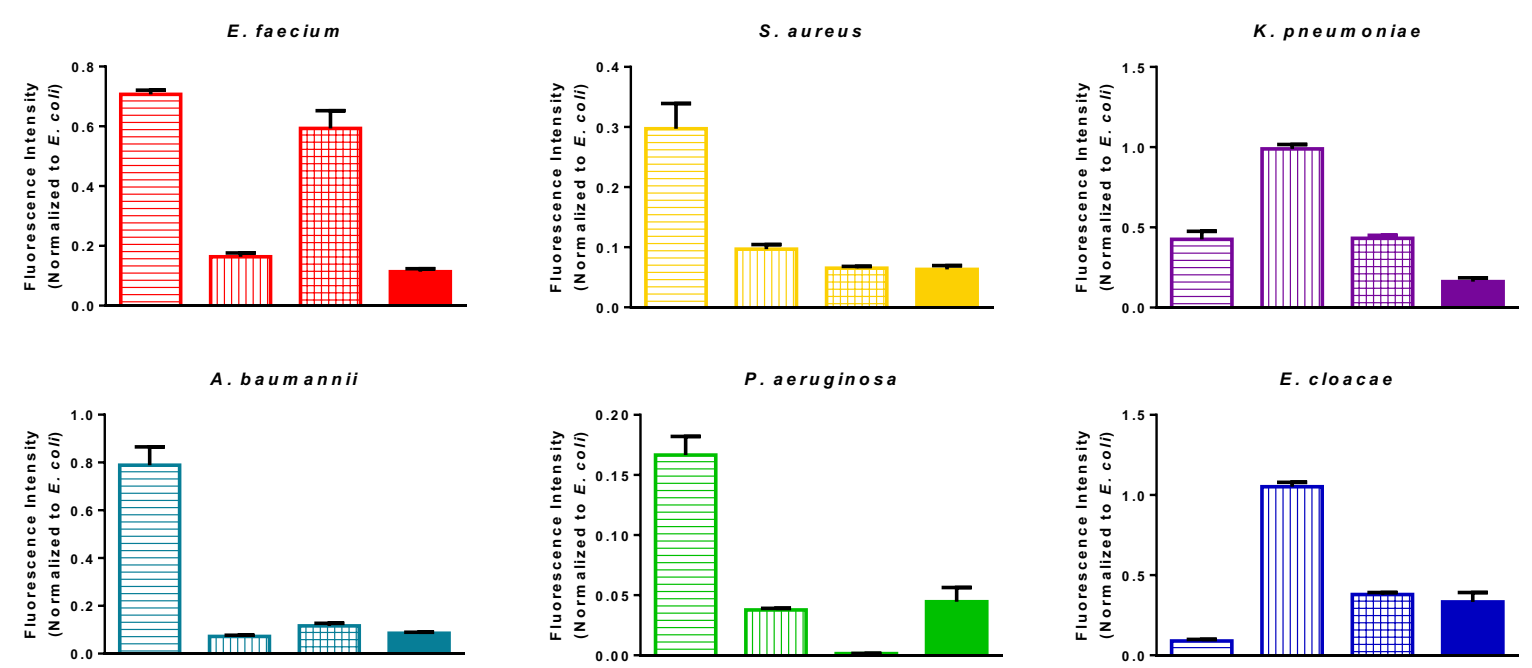

E. cloacae

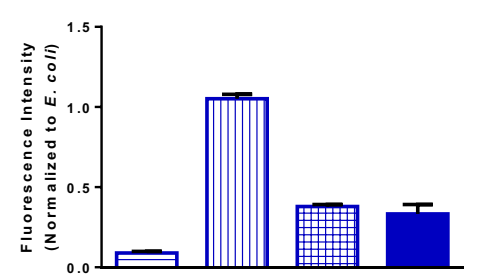

Figure 4: Quantification of in vitro trans-translation. Normalized fluorescence obtained in heterologous and homologous systems are shown at 310 minutes of incubation and reassembled by species. The results were normalized to the $E$. coli conditions and are shown as means \pm standard deviations.

The molecules being investigated for the development of new anti-trans-translation antibiotics will have different ways of interfering with tmRNA-SmpB binding to stalled ribosomes. They could disrupt tmRNA-SmpB interactions, or they could prevent interactions between the complex and the ribosome, such as by blocking the entrance of SmpB entirely or by preventing the passage of the complex through the bridges which have to be open during the process. Therefore, it is of great interest to have the ability to evaluate the targeting of the three main actors (tmRNA, SmpB, and the ribosome) independently as well as in each ESKAPE system. Of the 24 combinations we tested, 23 exhibited a signal strong enough for evaluating the possible activity of inhibitors. The only one that did not was the $P$. aeruginosa tmRNA-SmpB complex when used with $E$. coli ribosomes. We first suspected that the tmRNA H5 helix, inspired from the E. coli helix (Supp. Fig. 2B), might somehow have altered its activity. Therefore, to avoid any possible effects of the helical rearrangement, we constructed and tested

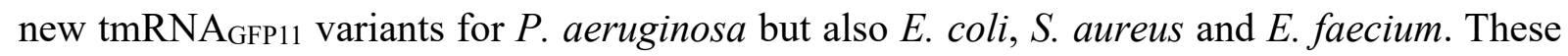


tmRNA ${ }_{\mathrm{GFP} 11} \mathrm{~V} 2$ constructs all have the full sequence that encodes the eleventh beta-strand of

GFP upstream from the natural H5 helix (Supp. Fig. 2C). However, these variants did not have improved fluorescence, and P. aeruginosa still did not emit signals. We also performed new experiments by increasing two fold the amounts of SmpB and two to four fold the amounts of tmRNA, but without further success (not shown). We can thus exclude the idea that the different structural features between the $P$. aeruginosa and E. coli ribosomes (Halfon et al., 2019) are important enough to prevent the correct process from occurring.

To permit the high-throughput screening of chemical compounds in multi-well microplates it was important to lower average screening costs of the current assay. To enable this, we decreased the reaction scale of the assays by reducing the final reaction volumes down to a microliter scale. Proof-of-concept experiments were performed with E. coli or ESKAPE homologous systems in final volumes of $2 \mu 1$ using the MANTIS ${ }^{\circledR}$ liquid-handler instrument (Formulatrix) or simply by using an electronic micropipette. The resulting signals were strong enough to allow for the easy detection of trans-translational activity. Indeed, the objective of this study was to create a non-hazardous in vitro screening system for evaluating transtranslation in ESKAPE pathogens, and to miniaturize it for HTS applications, and the assays we performed were convincing. We then decided to perform an experiment demonstrating proof of principle by using an oligonucleotide that interferes with the mRNA-like domain (MLD) of tmRNA as well as CT1-83 and KKL-35, two 1,3,4-oxadiazole derivatives that were recently shown to display a low in vitro activity against E. coli trans-translation (Guyomar et al., 2020). Towards this aim we used again the homologous system including tmRNA, SmpB and ribosomes from the same ESKAPE. The results on trans-translation show a total inhibition of the process when using the anti-sense, whatever the pathogen. On the other hand, and despite a very slight and dose-dependent effect of CT1-83 on P. aeruginosa and E. cloacae and KKL-35 on $S$. aureus, none of the compounds displayed any noticeable activity on the six ESKAPE 
systems (Figure 5A). Of course, to avoid compounds that inhibit any necessary step for fluorescence (e.g. transcription, translation, or GFP folding) to be scored as positive and result in false positive hits, transcription-translation assays were also performed using full GFP in $\mathrm{t}$ the absence of tmRNA-SmpB. This set of results confirmed the absence of noticeable effect of the two oxadiazole compounds on transcription-translation, while the signal was completely abolished after chloramphenicol treatment (Figure 5B). While this is interesting, it especially confirms that new classes of more efficient molecules are needed to target trans-translation in ESKAPE pathogens.

A Trans-translation
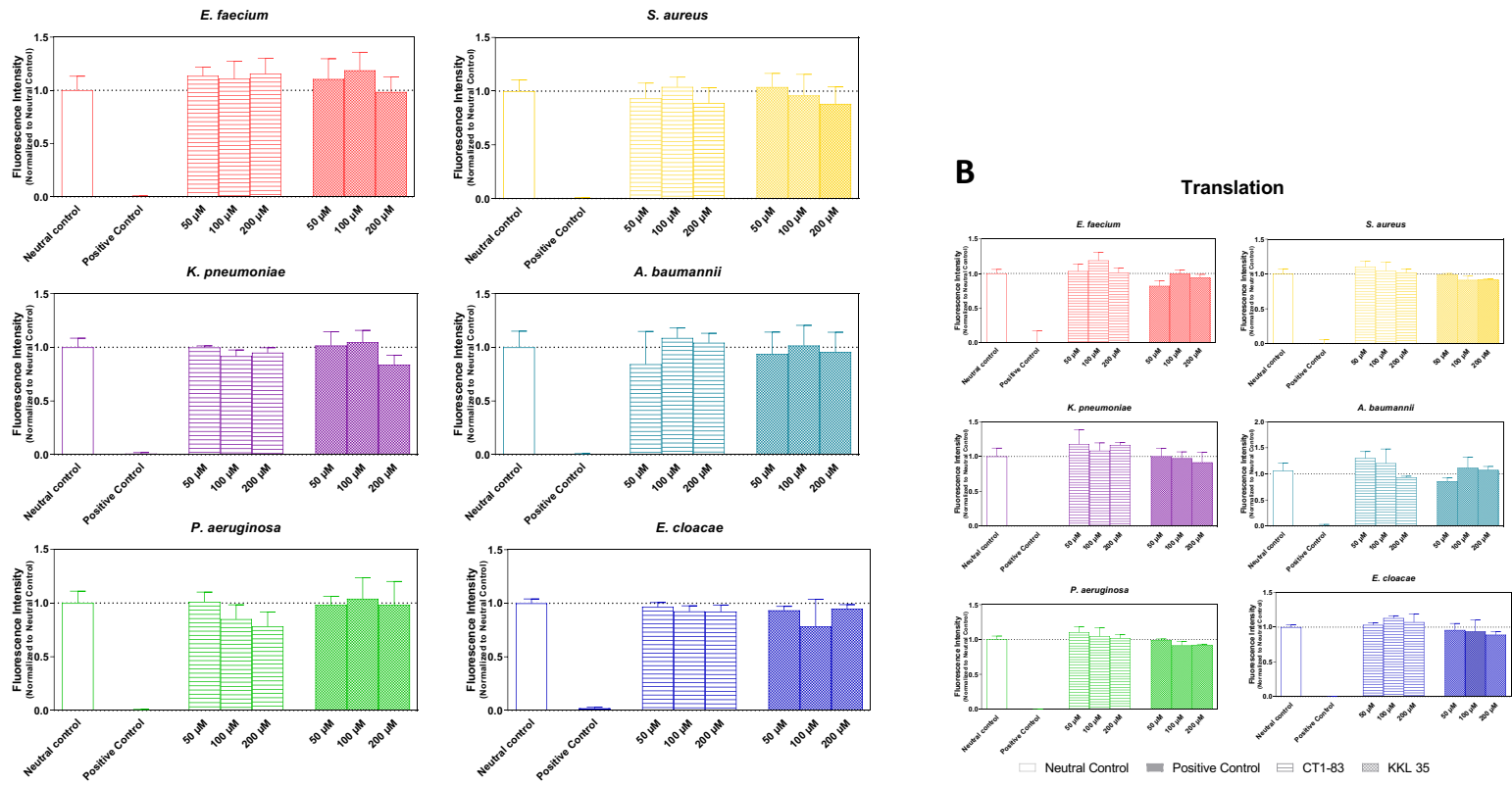

296

$\square$ Neutral control $\square$ Positive Control

$\boxminus \mathrm{CT} 1-83 \times \mathrm{KKL} 35$

Figure 5: Quantification of in vitro ESKAPE trans-translation and translation assays after CT1-

83 and KKL-35 treatment. Experiments were conducted in microplates, using ESKAPE homologous systems and increasing concentrations (50,100 and $200 \mu \mathrm{M})$ of CT1-83 and KKL-35 oxadiazole compounds. Normalized fluorescence intensities obtained are presented after 310 minutes of incubation and sorted by species. The results were normalized to the Neutral control conditions and are shown as means \pm standard deviations. A) Trans-translation assay with the oxadiazole compounds CT1-83 and KKL-35. The positive control was obtained by using $10 \mu \mathrm{M}$ Antisense B in $10 \%$ DMSO. B) 
Translation assay with the oxadiazole compounds CT1-83 and KKL-35. The positive control was obtained by using Chloramphenicol at $100 \mu \mathrm{M}$ in $10 \%$ DMSO. Using the Anova test, results were considered statistically significant when $\mathrm{P} \leq 0.01$. Only positive controls presented a difference of $\mathrm{P} \leq$ 0.0001 for translation and trans-translation (stars were not represented to facilitate graph reading).

Therefore, the system will clearly be very effective for benchmarking the effects of new antibiotic compounds that target trans-translation in highly pathogenic bacteria, as well as aiding us to better understand the trans-translation process in these bacteria. Its flexibility in the choice of target bacterial species and the possibility for varying the combinations of tmRNA, $\mathrm{SmpB}$, and ribosomes are advantageous, making the identification of new specific antimicrobial inhibitors easier. When hits are recovered it is important to perform a translation assay to confirm that the compounds do not inhibit any other step necessary for fluorescence (e.g. transcription, amino-acylation, translation, or GFP folding). Ongoing experiments in our laboratory are using this to screen large chemical and natural product libraries for drug discovery.

\section{Experimental Procedures}

\section{In silico analysis}

Complete genomes were retrieved from the NCBI database (March 2020). Chromosomes and plasmids (when present) were studied separately. GenBank files were first searched based on their textual annotation entries, using the keywords 'ArfA,' 'yhdL,' and 'alternative ribosomerescue factor' (for ArfA), or 'ArfB,' 'yaeJ,' 'ribosome-associated protein,' and 'peptidyl-tRNA hydrolase' (for ArfB). Missing loci were checked using BlastN, BlastP, and tBlastN similaritydetection strategies (Altschul et al., 1990) as well as comparative genomics, with synteny analysis done using progressiveMauve (Darling et al., 2010). All retrieved loci were compared using the Reciprocal Best Hits method, and InterProScan (Jones et al., 2014) was used on the 
corresponding proteins to check for the presence of the IPR005589/ PF03889 (ArfA) and IPR000352/PF00472 (ArfB) domains. Frameshifted loci were indicated as annotated in the GenBank files. Finally, the presence and absence of K09890 (ArfA) and K15034 (ArfB) were checked in the KEGG ORTHOLOGY database (Kanehisa et al., 2016).

\section{Plasmid construction and preparation}

For each ESKAPE tmRNA, the internal open reading frame was replaced by the eleventh betastrand of the superfolder GFP (sfGFP) preceded by the first conserved alanine of native tmRNA. In order to preserve the H5 helix, compensatory mutations were added (Fig. 2B). Additionally, the sequences were designed to carry a T7 promotor sequence in the 5'-end in order to realize transcription in vitro. Note that the tmRNA 3 '-end natural sequences from $E$. faecium (UUG) and S. aureus (UAU) were replaced by CCA so that the E. coli AlaRS could correctly aminoacylate them.

We also produced tmRNA ${ }_{\mathrm{GFP} 11} \mathrm{~V} 2$ variants for E. coli, P.aeruginosa, S.aureus and E. faecium species. This tmRNA ${ }_{\mathrm{GFP} 11}$ series carries the full sequence encoding the eleventh beta-strand of GFP upstream from the E. coli $\mathrm{H} 5$ helix (Supp. Fig. 2C). In order to obtain mature tmRNA ${ }_{\mathrm{GFP} 11}$ by in vitro transcription, the tmRNA $\mathrm{GFP} 11_{1}$ and tmRNA $\mathrm{GFP} 11_{\mathrm{V} 2}$ ESKAPE sequences were synthesized and cloned into the pUC19 vector between the HindIII and BamHI restriction sites (Supp. Table 1). For each ESKAPE SmpB, GenScript synthesized the sequences with codon optimization for E. coli, cloning them into the pET22b(+) vector between the NdeI and XhoI restriction sites to add a 6His histidine tag (Supp. Table 2). The generated plasmids, pUC19ESKAPEtmRNA GFP11 $_{1}$ and pET22b+ESKAPESmpB (Supp. Table 5), were amplified in E. coli NM522 cells then extracted using a NucleoBond Xtra Midi kit (Macherey-Nagel). Quantification was performed using a SimpliNano spectrophotometer (Biochrom).

\section{SmpB purification}


The bacterial cultures and SmpB purification were all done as previously described (Guyomar et al., 2020). His-tagged E. coli and ESKAPE SmpB proteins (Supp. Table 2) were expressed from the $\mathrm{pF} 1275$ and the $\mathrm{pET} 22 \mathrm{~b}+\mathrm{ESKAPE}$ SmpB vectors under the control of a T7 promoter in BL21(DE3) $4 s s r A$ cells (Cougot et al., 2014). Briefly, BL21(DE3) $\Delta s s r A$ cells were grown in lysogeny broth (LB) at $30{ }^{\circ} \mathrm{C}$ supplemented with ampicillin $(100 \mu \mathrm{g} / \mathrm{ml})$ and kanamycin $(50$ $\mu \mathrm{g} / \mathrm{ml})$. Protein expression was induced in the exponential phase $\left(O D_{600}=0.6\right)$ with $0.1 \mathrm{mM}$ isopropyl- $\beta$-D-1-thiogalactopyranoside (IPTG) overnight at $16{ }^{\circ} \mathrm{C}$. Cells were harvested and washed, then resuspended in lysis buffer $(50 \mathrm{mM}$ HEPES-KOH, $200 \mathrm{mM} \mathrm{KCl}, 20 \mathrm{mM}$ imidazole, and $1 \mathrm{mM}$ DTT pH 7.5). Cell lysis was performed using a French press, and the lysate was centrifuged at $15,000 \mathrm{rpm}$ for 45 minutes at $4{ }^{\circ} \mathrm{C}$ in a Beckman J2-MC with a JA-17 rotor. The supernatant was then filtrated $(0.2 \mu \mathrm{m})$ and injected onto a Ni-NTA sepharose column (HisTrap FF, GE Healthcare) previously equilibrated with the lysing buffer. The column was washed with $100 \mathrm{ml}$ lysis buffer and $50 \mathrm{ml}$ washing buffer (50 mM HEPES-KOH, $200 \mathrm{mM} \mathrm{KCl}, 1 \mathrm{M} \mathrm{NH}_{4} \mathrm{Cl}$, imidazole $20 \mathrm{mM}$, and $1 \mathrm{mM}$ DTT $\mathrm{pH}$ 7.5) before elution with 500 mM imidazole. Finally, a 10kDa Amicon Ultra centrifugal filter (Merck Millipore) was used to concentrate the fractions containing pure $\mathrm{SmpB}$, changing the buffer to a concentration buffer (50 mM HEPES-KOH, $100 \mathrm{mM} \mathrm{KCl,} \mathrm{10 \%} \mathrm{glycerol,} \mathrm{and} 1 \mathrm{mM}$ DTT pH 7.5). In order to visualize SmpB, 50 pmol of denatured proteins was analyzed on 15\% SDS-PAGE gels. Proteins were detected using InstantBlue protein stain (Expedeon) according to the supplier's instructions.

\section{tmRNAGFP11 production}

E. coli and ESKAPE tmRNA GFP11 $_{11}$ were produced as previously described (Guyomar et al., 2020). Each ESKAPE tmRNAGFP11 was transcribed in vitro from the pUC19ESKAPEtmRNA ${ }_{\text {GFP11 }}$ plasmids. To generate the 3' end needed for aminoacylation by AlaRS, the plasmid $(10 \mu \mathrm{g})$ was completely digested by NEB BsmBI or EarI restriction 
enzymes (Supp. Table 5). After phenol/chloroform extraction, the purified digested plasmid was precipitated, and the resulting pellets resuspended in $40 \mu 1$ nuclease-free water. A MEGAscript T7 transcription kit (Thermo Fisher Scientific) was used to produce each ESKAPE tmRNA $_{\text {GFP11 }}$ before its purification using the corresponding MEGAclear kit. Denatured tmRNA $_{\text {GFP11 }}$ was checked by electrophoresis on $8 \%$ Urea-PAGE gels, stained with ethidium bromide, and visualized under ultraviolet light.

\section{DNA templates and oligonucleotide production}

For trans-translation assays, the nonstop GFP1-10 sequence was produced by PCR using primers \#1 and 2 and Q5 High-Fidelity DNA Polymerase (NEB) with pETGFP 1-10 vector as a template (Cabantous et al., 2006; Supp. Table 3 and 4). For translation assays, primers \#1 and \#3 from the same template were used to amplify sfalaGFP, the superfolder GFP having an additional conserved alanine between the sfGFP1-10 and sfGFP11 beta-strands (Supp. Table 3 and 4). The resulting PCR products were purified using a QIAquick PCR Purification Kit (Qiagen) and checked by agarose electrophoresis. Both PCR products have a T7 promoter upstream from their coding sequences. Antisense oligonucleotide "A" was supplied by Eurofins (Supp. Table 3).

\section{ESKAPE ribosome purification}

Ribosomes were purified from Acinetobacter baumannii (clinical isolate); Staphylococcus aureus (clinical isolate); Pseudomonas aeruginosa (ATCC 27853); Enterobacter cloacae (clinical isolate); Klebsiella pneumoniae (clinical isolate); and Enterococcus faecium (HM1070). From an overnight starter culture, 6-9 L of LB medium were inoculated to reach an $\mathrm{OD}_{600}$ of 0.05 , then stirred at $150 \mathrm{rpm}$ at $37^{\circ} \mathrm{C}$. Bacterial growth was stopped when the $\mathrm{OD}_{600}$ reached 0.8 to 1.0 . The cells were then centrifuged at $4,000 \mathrm{rpm}$ for 20 minutes at $4{ }^{\circ} \mathrm{C}$. Pellets (around $2 \mathrm{~g} / \mathrm{L}$ of culture) were washed in a lysis buffer $(20 \mathrm{mM}$ Tris- $\mathrm{HCl} \mathrm{pH} \mathrm{7.5,} 20 \mathrm{mM} \mathrm{MgCl}$, $200 \mathrm{mM} \mathrm{NH}_{4} \mathrm{Cl}, 0.1 \mathrm{mM}$ EDTA, and $6 \mathrm{mM} \beta$-mercaptoethanol), centrifuged at 4,000 rpm for 
15 minutes at $4{ }^{\circ} \mathrm{C}$, and kept overnight at $-80{ }^{\circ} \mathrm{C}$. Pellets were then suspended in a Potter homogenizer in another lysis buffer complemented with $1 \mathrm{mM} \mathrm{CaCl}_{2}$. Cells were lysed in a French press at $1.0 \mathrm{kbar}$. To remove cellular debris, the lysates were centrifuged using a type $50.2 \mathrm{Ti}$ rotor at $18,200 \mathrm{rpm}$ for 30 minutes at $4{ }^{\circ} \mathrm{C}$. The superficial pellet layer was then discarded, and the pellet resuspended in lysis buffer. Ribosomes were isolated by centrifuging lysates on a $30 \%$ sucrose cushion at $31,500 \mathrm{rpm}$ for 19 hours at $4{ }^{\circ} \mathrm{C}$. The superficial layer of pellets was again discarded, leaving only the transparent pellets which were then resuspended in conservation buffer $\left(20 \mathrm{mM}\right.$ Tris- $\mathrm{HCl} \mathrm{pH} 7.5,10 \mathrm{mM} \mathrm{MgCl}_{2}, 50 \mathrm{mM} \mathrm{NH}_{4} \mathrm{Cl}, 0.1 \mathrm{mM}$ EDTA, and $6 \mathrm{mM} \beta$-mercaptoethanol). Any remaining contaminants were removed by a final centrifugation at 18,200 rpm for 1 hour at $4{ }^{\circ} \mathrm{C}$. Ribosomes were concentrated using a Centricon (Merck Millipore) with a cut-off of $100 \mathrm{~K}$, flash-frozen in nitrogen, and conserved at $-80^{\circ} \mathrm{C}$.

\section{Trans-translation assays}

In vitro trans-translation assays were performed using the PURExpress In Vitro Protein Synthesis and $\Delta$ Ribosome kits (New England Biolabs). For trans-translation assays, PURExpress was supplemented by $62.5 \mathrm{ng}$ purified PCR product encoding for nonstop sfGFP1$10,12.5$ pmol tmRNA ${ }_{\mathrm{GFP} 11}, 25 \mathrm{pmol} \mathrm{SmpB}$, and $50 \mathrm{pmol}$ antisense A. Where necessary $(\Delta$ Ribosome), 6.725 pmol ribosomes were also added. These reactions were performed in a final reaction volume of $10 \mu \mathrm{L}$, with PURExpress diluted by a final factor of 1.6 with Buffer III (HEPES-KOH 5mM pH7.5, MgOAc 9mM, NH4Cl 10mM, KCl 50mM, and DTT 1mM). A Step One Plus PCR system (Applied Biosystems) was used for incubation at $37{ }^{\circ} \mathrm{C}$ as well as for fluorescence measurements over 710 minutes.

\section{Translation assays}

In vitro translation assays were performed using a PURExpress $\Delta$ Ribosome kit. To produce the sfalaGFP, the PURExpress $\Delta$ Ribosome was diluted to a final factor of 1.6 with Buffer III, to which was added $62.5 \mathrm{ng}$ purified PCR product and $6.725 \mathrm{pmol}$ of the appropriate ribosomes 
in a final reaction volume of $10 \mu \mathrm{L}$. The translation reactions were incubated at $37{ }^{\circ} \mathrm{C}$, and fluorescence was measured over 710 minutes using a Step One Plus.

\section{Miniaturization of the trans-translation assays for HTS}

In vitro miniaturization of the trans-translation assays was performed using the PURExpress In Vitro Protein Synthesis $\Delta$ Ribosome kit (New England Biolabs). The mix was diluted by a factor of 1,6 after addition of $2,5 \mu \mathrm{M} \mathrm{SmpB}, 1,25 \mu \mathrm{M}$ tmRNAGFP11, $672,5 \mathrm{nM}$ ribosomes, $6,25 \mathrm{ng} / \mu \mathrm{L}$ of purified PCR product encoding for nonstop sfGFP1-10 and $5 \mu \mathrm{M}$ antisense A. $2 \mu \mathrm{L}$ of Neutral control (10\% DMSO), $2 \mu \mathrm{L}$ of Positive Control $(10 \mu \mathrm{M}$ Antisense B in 10\% DMSO) and compounds in 10\% DMSO were mixed together in a qPCR 96-well plate. CT1-83 oxadiazole compound was provided by Dr. Mickael JEAN (Univ. Rennes) and KKL35 by Sigma Chemicals, respectively. Compounds and controls were then dried in SpeedVac Concentrator before being resolubilized by adding $2 \mu \mathrm{L}$ of PURExpress Mix in the same plate. Incubation at $37^{\circ} \mathrm{C}$ and fluorescence measurements over 310 minutes were simultaneous performed thanks to Synergy HTX from BioteK. The intensities of GFP were measured with excitation filter at 485/20 and emission filter at 528/20. The gain used was 116 for E. faecium, S. aureus, A. baumannii and P. aeruginosa and 100 for K. pneumoniae and E. cloacae.

The transcription-translation control assays were performed in the same way, except that nonstop sfGFP1-10 was replaced by full sfGFP, in the absence of tmRNA and SmpB. The Positive Control was then Chloramphenicol at $100 \mu \mathrm{M}$ in $10 \%$ DMSO.

\section{Acknowledgments}

The authors are particularly grateful to Axel Innis and Anne-Xander van der Stel for sharing their Mantis experiment expertise and to Charlotte Guyomar and Emmanuel Giudice for insightful comments on the manuscript. We thank Sylvie Georgeault and Fanny Demay for their 
help in purifying E. faecium, S.aureus and A. baumanni ribosomes and to Mickael Jean for providing CT1-83 oxadiazole compound.

Funding and additional information

This work was supported by the Agence Nationale pour la Recherche as part of the EU's Joint Programming Initiative on Antimicrobial Resistance project (JPIAMR) project "Ribotarget Development of novel ribosome-targeting antibiotics" as well as by SATT Ouest-Valorisation (DV 2552 and DV 3506).

Author contributions

M.T. cloned and purified the SmpB and tmRNA variants and performed and analyzed the transtranslation in vitro assays. R.C.D.S. performed translation and trans-translation in vitro assays. E.V. purified ribosomes from A. baumannii, E. cloacae, K. pneumoniae, P. aeruginosa, and $S$. aureus. F.B.H. performed in silico analysis of ArfA and ArfB in bacterial genomes. R.G. designed the study. E.E., D.B., and R.G. supervised the project. M.T. and R.G. wrote the manuscript, and all authors approved its final version.

\section{Conflict of interest}

Reynald Gillet is co-inventor of the system described here (patent application \#EP/2018/063780).

\section{References}

Altschul, S.F., Gish, W., Miller, W., Myers E.W. and Lipman, D.J. (1990) Basic Local Alignment Search Tool. J. Mol. Biol. 215, 403-410. 
Barends, S., Wower, J., Kraal, B. (2000) Kinetic parameters for tmRNA binding to alanyltRNA synthetase and elongation factor $\mathrm{Tu}$ from Escherichia coli. Biochemistry. 39(10):2652-8.

Brunel,R., Descours,G., Durieux,I., Doublet,P., Jarraud,S. and Charpentier,X. (2018) KKL35 Exhibits Potent Antibiotic Activity against Legionella Species Independently of transTranslation Inhibition. Antimicrob. Agents Chemother. 62, e01459-17.

Cabantous,S. and Waldo,G.S. (2006) In vivo and in vitro protein solubility assays using split GFP. Nat. Methods, 3, 845-85

Cougot,N., Molza,A.-E., Delesques,J., Giudice,E., Cavalier,A., Rolland,J.-P., Ermel,G., Blanco,C., Thomas,D. and Gillet,R. (2014) Visualizing compaction of polysomes in bacteria. J. Mol. Biol., 426,377-388.

Darling, A.E., Mau, B., and Perna N.T. (2010) progressiveMauve: multiple genome alignment with gene gain, loss and rearrangement. PLoS One. 5(6):e11147

Dillon, N.A., Peterson, N.D., Feaga, H.A., Keiler, K.C., Baughn, A.D. (2017) Antitubercular Activity of Pyrazinamide is Independent of trans-Translation and RpsA. Sci. Rep. 7,6135 .

Giudice E, Macé K, Gillet R. (2014) Trans-translation exposed: understanding the structures and functions of tmRNA-SmpB. Front Microbiol. 5,113.

Goralski, T.D.P., Kirimanjeswara, G.S., Keiler, K.C. (2018) A New Mechanism for Ribosome Rescue Can Recruit RF1 or RF2 to Nonstop Ribosomes. mBio. 9, e02436-18.

Guyomar, C., Thépaut, M., Nonin-Lecomte, S., Méreau, A., Goude, R., Gillet, R. (2020) Reassembling green fluorescent protein for in vitro evaluation of trans-translation. Nucleic Acids Res. 48(4):e22.

Halfon, Y., Jimenez-Fernandez, A., La Rosa, R., Espinosa Portero, R., Krogh Johansen, H., Matzov, D., Eyal, Z., Bashan, A., Zimmerman, E., Belousoff, M., Molin, S., Yonath, A. (2019) Structure of Pseudomonas aeruginosa ribosomes from an aminoglycoside-resistant clinical isolate. Proc. Natl. Acad. Sci. U S A. 116, 22275-22281.

Himeno, H., Nameki, N., Kurita, D., Muto, A., Abo, T. (2015) Ribosome rescue systems in bacteria. Biochimie. 114, 102-12.

Hudson, C.M., and Williams, K.P. (2015) The tmRNA website. Nucleic Acids Res. 43 (Database issue):D138-40.

Jones, P., Binns, D., Chang, H.Y., Fraser, M., Li, W., McAnulla, C., McWilliam, H., Maslen, J., Mitchell, A., Nuka, G., Pesseat, S., Quinn, A.F., Sangrador-Vegas, A., Scheremetjew, M., Yong, S.Y., Lopez, R. and Hunter, S. (2014). InterProScan 5: genomescale protein function classification. Bioinformatics. 30, 1236-1240.

Kanehisa, M., Sato, Y., Kawashima, M., Furumichi, M. and Tanabe, M. (2016) KEGG as a reference resource for gene and protein annotation. Nucleic Acids Res. 44(D1):D457-62.

Karzai, A.W., Susskind, M.M., Sauer, R.T. SmpB, a unique RNA-binding protein essential for the peptide-tagging activity of SsrA (tmRNA). (1999) EMBO J. 18:3793-9. 
Keiler, K.C., and Feaga, H.A. Resolving nonstop translation complexes is a matter of life or death. (2014) J Bacteriol. 196, 2123-30.

Li, J., Ji, L., Shi, W., Xie, J., Zhang, Y. (2013) Trans-translation mediates tolerance to multiple antibiotics and stresses in Escherichia coli. J. Antimicrob. Chemother. 68, 247781.

Liu, P., Chen, Y., Wang, D., Tang, Y., Tang, H., Song, H., Sun, Q., Zhang, Y., Liu, Z. (2016) Genetic Selection of Peptide Aptamers That Interact and Inhibit Both Small Protein B and Alternative Ribosome-Rescue Factor A of Aeromonas veronii C4. Front. Microbiol. 7,1228 .

Lytvynenko, I., Paternoga H., Thrun, A., Balke, A., Müller, T.A., Chiang, C.H., Nagler, K., Tsaprailis, G., Anders, S ., Bischofs, I., Maupin-Furlow, J.A., Spahn, C.M.T., Joazeiro, C.A.P. (2019) Alanine Tails Signal Proteolysis in Bacterial Ribosome-Associated Quality Control. Cell. 178(1):76-90.

Macé,K., Demay,F., Guyomar,C., Georgeault,S., Giudice,E., Goude,R., Trautwetter,A., Ermel,G., Blanco,C. and Gillet,R. (2017) A Genetic Tool to Quantify trans-Translation Activity in Vivo. J. Mol. Biol., 429, 3617-3625.

Müller C., Crowe-McAuliffe, C. and Wilson D.N. (2021) Ribosome Rescue Pathways in

Osterman, I.A., Bogdanov, A.A., Dontsova, O.A., Sergiev, P.V. (2016) Techniques for Screening Translation Inhibitors. Antibiotics (Basel). 5

Ramadoss, N.S., Alumasa, J.N., Cheng, L., Wang, Y., Li, S., Chambers, B.S., Chang, H., Chatter-jee, A.K., Brinker, A., Engels, I.H., et al. (2013) Small molecule inhibitors of transtranslation have broad-spectrum antibiotic activity. Proc. Natl. Acad. Sci. U. S. A., 110, 10282-10287.

Rice, L. B. (2008) Federal funding for the study of antimicrobial resistance in nosocomial pathogens: no ESKAPE. J. Infect. Dis. 197, 1079-1081

Santajit, S., Indrawattana, N. (2016) Mechanisms of Antimicrobial Resistance in ESKAPE Pathogens. Biomed Res Int. 2475067

Shi, W., Zhang, X., Jiang, X., Yuan, H., Lee, J.S., Barry, C.E. 3rd, Wang, H., Zhang, W., Zhang, Y. (2011) Pyrazinamide inhibits trans-translation in Mycobacterium tuberculosis. Science. 333, 1630-2.

Schönhuber, W., Le Bourhis, G., Tremblay, J., Amann, R., Kulakauskas, S. (2001). Utilization of tmRNA sequences for bacterial identification. BMC Microbiol. 1, 20.

Shimizu,Y., Inoue,A., Tomari,Y., Suzuki,T., Yokogawa,T., Nishikawa,K., Ueda,T. (2001) Cell-free translation reconstituted with purified components. Nat. Biotechnol., 19, 751-755.

Shimizu, Y., Ueda, T. The role of SmpB protein in trans-translation. (2002) FEBS Lett.514, 74-7. 

S. (2019) Release factor-dependent ribosome rescue by BrfA in the Gram-positive bacterium Bacillus subtilis. Nat. Commun. 10:5397. guide research, discovery, and development of new antibiotics World Health Organization. 